\title{
环境因素对天目山柳杉树轮 $\boldsymbol{\delta}^{13} \mathrm{C}$ 方位分布的影响
}

\author{
邓自旺 ${ }^{12}$ 钱君龙 ${ }^{3}$ 屠其璞 $^{2}$ 湩培民 $^{3}$ 黄纯朴 $^{3}$ \\ 柯善哲 ${ }^{4}$ 黄耀生 ${ }^{4}$ 唐劲松 ${ }^{3}$ 柯晓康 ${ }^{4}$ \\ (1 南京师范大学地学院, 南京 210093) (2 南京气象学院资源环境系, 南京 210044) \\ (3 中国科学院南京地理与湖泊研究所, 南京 210008) (4 南京大学地球科学系, 南京 210093)
}

\begin{abstract}
摘 要 应用方差分析、经验正交函数、合成分析, 研究了天目山柳杉 (Cryptomeria fortunei) 树轮中 $\delta^{13} \mathrm{C}$ 随方位的分 布特征。应用相关分析探讨了这种分布特征的年际差异与环境因素的关系。结果表明 树轮 $\delta^{13} \mathrm{C}$ 值有显著方位差 异和年际变化, 不同方位的差异可以用 4 个基本角分布型表示, 其中一型表示各方位 $\delta^{13} \mathrm{C}$ 比多年平均值偏大, 其中 北 $(\mathrm{N})$ 、西 (W)、西南 $(\mathrm{SW}) 、$ 南 $(\mathrm{S})$ 较平均值偏大约 0.5 , 而其余 4 个方位偏大不到 0.2 二型各方位也较多年平均值偏 大, 但各方位差异不大, 除 $\mathrm{N}$ 方位较小外, 其余均在 $0.2 \sim 0.4$ 之间。三型为各方位 ${ }^{13} \mathrm{C}$ 较平均状况偏小, 其中西北

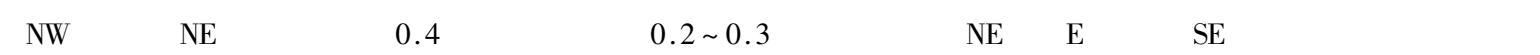
(NW) 方位较平均状况偏大约 $0.1, \mathrm{~N}$ 方位偏小 0.2 其余 3 个方位则偏小约 0.3 。而其年际变化既可用这 4 个角分 布型的更替表示, 也可以用第一、二主成分表示。 $\delta^{13} \mathrm{C}$ 的第一主成分与前一年 $8 \sim 12$ 月降水量负相关, 与当年 $1 \sim 3$ 月降水正相关。第二主成分与前一年 8 月至当年 1 月的 6 个月平均气温正相关。不同的角分布型与不同的气候状 态相联系。一型出现的有利气候条件是 $: \mathrm{R}_{8 \sim 12}$ 少、 $\mathrm{R}_{1 \sim 3}$ 多、 $\mathrm{T}_{3 \sim 7}$ 低并且 $\mathrm{T}_{8 \sim 1}$ 高; 二型出现的有利气候条件是 $: \mathrm{R}_{8 \sim 12}$ 少、 $\mathrm{R}_{1 \sim 3}$ 多、 $\mathrm{T}_{3 \sim 7}$ 低并且 $\mathrm{T}_{8 \sim 1}$ 低:三型出现的有利气候条件是 $: \mathrm{R}_{8 \sim 12}$ 多、 $\mathrm{R}_{1 \sim 3}$ 少、 $\mathrm{T}_{3 \sim 7}$ 高并且 $\mathrm{T}_{8 \sim 1}$ 高; 四型出现的有利 气候条件是 $: R_{8 \sim 12}$ 多、 $R_{1 \sim 3}$ 少、 $T_{3}$ 高并且 $\mathrm{T}_{8 \sim 1}$ 低。可见角分布型的差异可能包含着环境变化的信息, 因此同时利 用树轮整体平均 $\delta^{13} \mathrm{C}$ 值的序列和角分布型序列来重建历史气候, 可以得到更多的历史气候变化信息。
\end{abstract}

关键词 环境因素 柳杉(Cryptomeria fortunei) 树轮 $\delta^{13} \mathrm{C} \quad$ 角分布

\section{EFFECTS OF ENVIRONMENTAL FACTORS ON $\delta{ }^{13} \mathrm{C}$ AZIMUTH DISTRIBUTION IN CRYPTOMERIA FORTUNEI TREE RINGS IN TIANMU MOUNTAIN REGIONS}

\author{
DENG Zi-Wang $^{12}$ QIAN Jun-Long ${ }^{3} \quad$ TU Qi-Pu ${ }^{2}$ PU Pei-Min ${ }^{3} \quad$ HUANG Chun-Pu ${ }^{3}$ \\ KE Shan-Zhe ${ }^{4}$ HUANG Yao-Sheng ${ }^{4}$ TANG Jin-Song ${ }^{3}$ and KE Xiao-Kang ${ }^{4}$ \\ (1 Institute of Geography, Nanjing Normal University, Nanjing 210093 , China) \\ (2 Department of Resource and Environment, Nanjing Institute of Meteorology, Nanjing 210044 , China) \\ (3 Nanjing Institute of Geography and Limnology, the Chinese Academy of Sciences , Nanjing 210008 , China) \\ (4 Department of Earth Sciences , Nanjing University , Nanjing 210093 , China)
}

\begin{abstract}
The characteristics of azimuth distribution of $\delta{ }^{13} \mathrm{C}$ in Cryptomeria fortunei tree rings in Tianmu Mountain regions was studied by means of statistic methods including variance analysis, composition analysis and empirical orthogonal function (EOF) analysis. The effects of environmental factors on the interannual alternation of $\delta{ }^{13} \mathrm{C}$ azimuth distribution patterns were also investigated by correlation analysis. The results showed that $\delta{ }^{13} \mathrm{C}$ in Cryptomeria tree rings varied along with both azimuth and time significantly. The variation along with azimuth can be represented by four basic azimuth distribution types (denoted by Types $1,2,3$ and 4). Type 1 : the $\delta{ }^{13} \mathrm{C}$ anomalies in all azimuths are positive, and they are $>0.5$ in north $(\mathrm{N})$, west $(\mathrm{W})$, southwest (SW) and south (S) and between 0.2-0.4 in the other four directions. Type 2 : also with positive anomalies in all azimuths as in Type 1 , but the $\delta{ }^{13} \mathrm{C}$ anomalies are in the interval 0.2-0.4 in most directions except for $\mathrm{N}(<0.2)$. Type 3 : the $\delta{ }^{13} \mathrm{C}$ anomalies are about -0.4 in the $\mathrm{NW}$ and $\mathrm{NE}$, and in the range of -0.3 to -0.2 in all other directions. Type $4:$ the anomalies in NE, E and SE directions are near 0.0 while at 0.1 , in the $\mathrm{NW},-0.2$ in $\mathrm{N}$, and -0.3 in the other 3 directions. The $\delta{ }^{13} \mathrm{C}$ variation with time can be presented by either the alternation of the four basic types or by the first two principle components (PC1 and
\end{abstract}


PC2) of the eight-azimuth $\delta{ }^{13} \mathrm{C}$ time-series. Furthermore, the PC1 significantly positively correlated with the total precipitation for the period from January to March and negatively correlated with the total rainfall for the period from August to December of the last year. There was a positive correlation between average temperature for the period from August to January along the PC2 axis. The ${ }^{13} \mathrm{C}$ azimuth distribution type varies under different climatic conditions. The favorable environmental factors for types 1 and 2 include : 1) shortage of precipitation from August to December of the preceding year ;2) richness of rainfall from January to March , and 3 ) lower temperature from March to July and higher (lower) temperature from last August to present January. The opposite climatic conditions were favorable to types 4 and 3 . This phenomenon suggests that the $\delta{ }^{13} \mathrm{C}$ azimuth distribution types may contain environmental information, which could be used to established more detailed historical climate data by both averaged $\delta^{13} \mathrm{C}$ in whole tree ring and its azimuth distribution type time series .

Key words Environmental factor, $\delta^{13} \mathrm{C}$, Cryptomeria fortunei , Tree ring , Azimuth distribution

许多研究表明,树轮宽度以及树轮中化学成分 含量对环境变化反应敏感(梁尔源等, 2001; 钱君龙 等 $2001 \mathrm{a} ; 2001 \mathrm{~b})$ 。树木生长过程中储存的碳稳定 性同位素组成可以反映大气中的 ${ }^{13} \mathrm{C} /{ }^{12} \mathrm{C}$ 变化和树 木对环境变化的生理响应(刘禹等, 1996 蒋高明等， $1997) 。{ }^{13} \mathrm{C} /{ }^{12} \mathrm{C}$ 比可以用 $\delta^{13} \mathrm{C}(\% o)$ 表示。一般而言， $\delta^{13} \mathrm{C}$ 值的大小与环境中 $\mathrm{CO}_{2}$ 同位素的组成、一年中 光合作用的时间、气候因子和其它一些潜在的生长 因子有关 (钱君龙等,2001a； Pieter \& Mook,1980)。 环境变量通过影响同化作用来影响 $\delta^{13} \mathrm{C}$, 其中温度 是通过影响光合作用速度对 $\delta^{13} \mathrm{C}$ 产生影响 (Beerling, 1996)或间接地通过土壤水分或湿度来影响 $\delta^{13}$ $\mathrm{C}$ (Schleser ,1995)。因此, 许多树轮研究者利用 $\delta^{13} \mathrm{C}$ 资料来对当地过去的气候状况进行估计 (Sheu \& Chiu，1995;Schleser et al. ,1999)。有些研究者已注 意到在年轮不同方位上春 (早)材/秋 (晚)材中的 $\delta^{13}$ C 常有 1\%o 4\%o的变化(Pieter \& Mook, 1980 :Schleser et al. 1999), 并认为这可能是因为树木不同根系得 到不同的水肥供应,进而影响与之相应部分树轮的 化学成分，还可能是因为不同方位树枝所接受的太 阳辐射不同造成的(Pieter \& Mook ,1980)。树轮 $\delta^{13} \mathrm{C}$ 在不同方位的差异(角分布)也存在年际变化, 即不 同年份各方位 $\delta^{13} \mathrm{C}$ 并不是同时系统地增大或系统 地减小, 而是存在明显的差异。例如有些方位增大， 而另一些方位则减小。以往的树轮 $\delta^{13} \mathrm{C}$ 研究侧重 于每年整个年轮 $\delta^{13} \mathrm{C}$ 平均值与环境变量之间的关 系,而对树轮 $\delta^{13} \mathrm{C}$ 角分布年际变化的研究则不多 见。对于一棵生长在自然状态下的树木而言, 其根 系的空间分布、土壤的化学成分、树冠的整体形状是 相对稳定的, 而变化最大的可能就是气候。那么 树 轮 $\delta^{13} \mathrm{C}$ 角分布的年际变化是由于气候的年际变化 引起的, 还是其它随机因素引起的? 假如是气候变 化引起的, 与哪些气候因子关系密切? 这是本文所 尝试探讨的问题。浙江天目山地处亚热带北缘, 属
亚热带季风性暖湿型气候, 四季变化明显, 雨量充 沛, 有利于各种植物生长, 其中有树龄为几百年的柳 杉, 这为本研究提供了客观条件。

\section{1 资料与方法}

\section{1 研究材料}

柳杉 (Cryptomeria fortunei $)$ 是天目山地区的代表 树种, 因此本工作选择了西天目山林区 $\left(32^{\circ} 20^{\prime} \mathrm{N}\right.$, $119^{\circ} 26^{\prime} \mathrm{E}$, 海拔 $1020 \mathrm{~m}$ ) 柳杉树轮为研究对象。为 了对年轮准确定年, 样本收集按国际树轮数据库标 准进行。采集了 20 株柳杉的生长雉 40 芯, 用以建 立该地区的树轮标准宽度年表。柳杉树盘取自一棵 1997 年 10 月被台风刮倒的柳杉树，树龄为 176 年， 离根 $50 \mathrm{~cm}$ 。树轮有明显的偏心, 其中 1996 年形成 的年轮在北、东、南、西方位上离中心的距离分别为 $180 、 195 、 450 、 386 \mathrm{~mm}$ 。由交叉定年技术确定年代。 在圆盘的 8 个方位上和覆盖整个年轮均匀地分别逐 年雕刻采集木质样 $2 \mathrm{~g}$ 左右。样品在 $70 \sim 80{ }^{\circ} \mathrm{C}$ 干燥 3 昼夜, 磨至 $30 \sim 60$ 目; 通过有机溶剂抽提, 清除全 部类脂物质 然后通过氯化作用和碱洗过程, 提出 $\alpha-$ 纤维素, 气体提取和质谱仪分析在南京大学地球科 学系成矿作用国家重点实验室完成:提取的 $\alpha$-纤维 素与 $\mathrm{CuO} 、 \mathrm{Pt}$ 丝在真空下密封于小石英管中, 高温燃 烧产生供质谱仪分析的 $\mathrm{CO}_{2}$ 气体。最后，将收集到 的 $\mathrm{CO}_{2}$ 在 MAT-251 质谱仪 (德国制造) 上分析测得 树轮 ${ }^{13} \mathrm{C} /{ }^{12} \mathrm{C}$ 同位素比值, 并通过换算以 $\delta^{13} \mathrm{C}_{\mathrm{PDB}}$ (简 称 $\delta^{13} \mathrm{C}$ ) 表示, 整个流程的分析误差 $\leqslant 0.2 \%$ 。

环境资料为天目山气象站 1976 1996 年的逐 月降水量、月平均气温、月平均最高气温、月平均最 低气温。

\section{2 方差分析}

为了分析树轮 $\delta^{13} \mathrm{C}$ 随方位变化的现象及其年 际变化的客观存在性, 采用了方差分析方法, 用以检 验几个总体平均值是否相等的问题 (屠其璞等, 
1984)。记历年各方位 $\delta^{13} \mathrm{C}$ 值为 $y_{k t}(k=1,2, \cdots, 8 ; t$ $=1,2, \cdots, 20), k$ 为方位序号, $t$ 为年序号。 $S_{1}$ 为 $\delta^{13} \mathrm{C}$ 各方位内部离差平方和, $S_{2}$ 为各方位的平均值 对总体平均值的离差平方和, $S_{3}$ 为 $\delta^{13} \mathrm{C}$ 各年内部离 差平方和 即

$$
\begin{aligned}
& S_{1}=\sum_{k=1}^{8} \sum_{t=1}^{20}\left(y_{k t}-\bar{y}_{k}\right)^{2} \\
& S_{2}=\sum_{k=1}^{8} 20\left(\bar{y}_{k}-\bar{y}_{\cdot} .\right)^{2} \\
& S_{3}=\sum_{k=1}^{8} \sum_{t=1}^{20}\left(y_{k t}-\bar{y}_{t}\right)^{2}
\end{aligned}
$$

式中 $\bar{y}_{k}=\frac{1}{20} \sum_{t=1}^{20} y_{k t}, \bar{y}_{\cdot t}=\frac{1}{8} \sum_{k=1}^{8} y_{k t}$ 和 $\bar{y} .=\frac{1}{160} \sum_{k=1}^{8} \sum_{t=1}^{20} y_{k t}$ 分别为各方位 $\delta^{13} \mathrm{C}$ 的多年平均值、各年 $\delta^{13} \mathrm{C}$ 多方位 的平均值和历年所有方位 $\delta^{13} \mathrm{C}$ 的总平均值。统计

$$
\begin{aligned}
& F_{1}=\frac{S_{2} / 7}{S_{1} / 152} \\
& F_{2}=\frac{S_{3} / 19}{S_{1} / 140}
\end{aligned}
$$

分别服从自由度为 $(7,152)$ 和 $(19,140)$ 的 $F$ 分布。 设信度 $\alpha=0.01$, 通过查表可得到临界值 $F_{\alpha}$ 分别为 2.76 和 2.03 , 当通过资料计算的 $F_{1}>2.76$ 时, 说明 $\delta^{13} \mathrm{C}$ 在不同方位存在着显著的差异。当 $F_{2}>2.03$ 时, 说明 $\delta^{13} \mathrm{C}$ 的方位差异存在显著的年际变化。

\section{3 经验正交函数展开分析 $(\mathrm{EOF})$}

为了同时研究 $\delta^{13} \mathrm{C}$ 随时间和方位的变化特征, 在本文中采用 $\mathrm{EOF}$ 对树轮 $\delta^{13} \mathrm{C}$ 资料矩阵进行分析 (屠其璞等, 1984)。所谓 EOF 就是将资料矩阵分解 成正交的时间函数与正交的空间 (本文为方位) 函数 乘积之和，即：

$$
y_{k t}=\sum_{h=1}^{8} T_{h t} X_{h k}
$$

式中 $X_{h k}(h=1,2, \cdots, 8)$ 为某种方位函数 (即仅随方 位而变化的函数)，反映了 $\delta^{13} \mathrm{C}$ 随方位变化的特征， 称为典型场 (典型角分布)。 $T_{h t}$ 为某种时间函数, 反 映了第 $h$ 典型场的权重系数(主成分)。共可得到 8 个典型角分布及与其对应的 8 个主成分。在实际分 析中前 $n$ 个典型场及其对应的 $n$ 个主成分就足以 反映分析对象的主要时空特征，一般以累计方差贡 献率达到 $85 \%$ 为标准 (本文中 $n=2$ )。将剩余的总 方差贡献率 $\leqslant 15 \%$ 的特征角分布和主成分 (本文为 6 个)作为随机因素引起的时空变化而忽略不记。 这样既达到了提取主要变化特征的目的, 又达到了 消除时空变化噪声的目的。然后根据两个主成分各 年对应值的正负号, 按年份进行分类, 即可将角分布
相似的年份归为同一类，而将角分布差异明显的年 份归为不同的类，达到分类的目的。通过对不同类 型年份角分布进行合成, 就可以得到几种基本角分 布类型 (本文共分 4 类)。

\section{4 相关分析}

相关分析是研究两个变量之间相关程度的一种 常用的分析方法，通过计算两变量之间线性相关系 数, 依据该系数绝对值的大小及符号, 判断二者之间 关系的密切程度。具体计算方法如下：

设 $x_{i}, y_{i}$ 为两个长度为 $n$ 的序列, 其平均值分 别为 $\bar{x}$ 和 $\bar{y}$ 则相关系数 $r$ 的计算公式如下:

$$
r=\frac{\sum_{i=1}^{n}\left(x_{i}-\bar{x}\right)\left(y_{i}-\bar{y}\right)}{\sqrt{\sum_{i=1}^{n}\left(x_{i}-\bar{x}\right)^{2}} \sqrt{\sum_{i=1}^{n}\left(y_{i}-\bar{y}\right)^{2}}}
$$

\section{$2 \delta^{13} \mathrm{C}$ 随方位、时间的变化及其与环境变量 的相关性}

\section{$2.1 \delta^{13} \mathrm{C}$ 随方位的变化}

对各方位 $\delta^{13} \mathrm{C}$ 作方差分析，通过公式(1) (3) 计算得 $S_{1}=24.8, S_{2}=23.77, F_{1}=20.82>2.76$, 不 同方位 $\delta^{13} \mathrm{C}$ 均值间的差异远远超过了信度 $\alpha=0.01$ 时的显著标准, 可见天目山柳杉树轮中 $\delta^{13} \mathrm{C}$ 在不同 的方位有显著的差异 (角分布)。通过对近 20 年资 料的平均, 发现极大值出现在北 (N) 方位, 为 -22.935 极小值出现在西 (W) 方位, 为 -24.112 (图 1)。其它 6 个方位从大到小依次为:西北 (NW) $(-23.044)$ 、东北 $(\mathrm{NE})(-23.14)$ 、东 $(\mathrm{E})(-23$. $311)$ 、东南 $(\mathrm{SE})(-23.313)$ 、南 $(\mathrm{S})(-23.657)$ 、西南 $(\mathrm{SW})(-23.839)$ 。除西北方向 $(\mathrm{NW})$ 外，其它 7 个方 位从 $\mathrm{N}$ 到 $\mathrm{W}$ 是按顺时针方向依次减小 (图 1)。这 种平均状态的角分布可能与树轮偏心等现象相似, 是由于树木生长环境中相对稳定的因素在各方位上 的差异造成的(Pieter \& Mook ,1980)。

\section{$2.2 \delta^{13} \mathrm{C}$ 角分布的年际变化特征}

通过公式 (1)、(3)、(5) 对资料计算, 得 $F_{2}=$ $9.233>2.03$, 即 $\delta^{13} \mathrm{C}$ 角分布年际变化通过了信度为 0.01 的显著性检验，表明 $\delta^{13} \mathrm{C}$ 角分布存在显著的年 际变化。年际变化中不但包含 8 个方位 $\delta^{13} \mathrm{C}$ 平均 值的变化, 也包括 $\delta^{13} \mathrm{C}$ 在各方位分布状态的变化。 由图 2 可见, 虽然年际差异很大(如 1994、1995 年与 相邻年相差很多), 但近 19 年来 $\delta^{13} \mathrm{C}$ 总体上是增大 的。

通过 $\mathrm{EOF}$ 分析, 可得到 8 个典型角分布及与之 


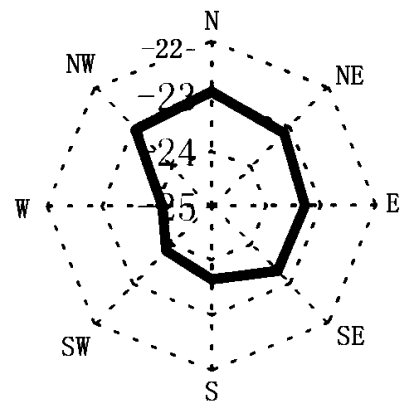

图 1 树轮 $\delta^{13} \mathrm{C} 19$ 年总体平均值随方位的变化

Fig. 1 The average $\delta^{13} \mathrm{C}$ in tree ring in the period of 19 years against azimuths

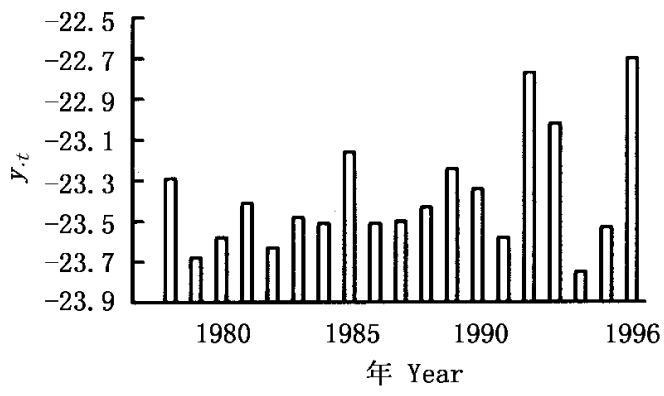

图 2 树轮 $\delta^{13} \mathrm{C} 8$ 个方位总体平均值 $\mathrm{y}$ 的年际变化

Fig. 2 The average $\delta^{13} \mathrm{C}$ in the eight sections of every tree ring $(y, t)$ against years
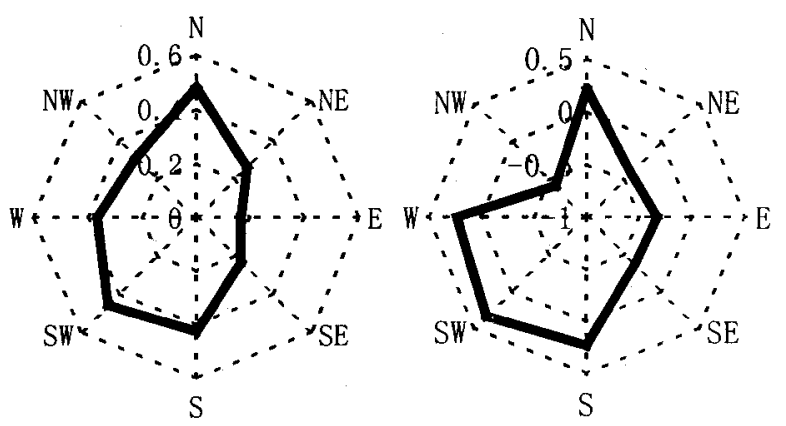

第一典型场

The first eigenvector

第二典型场

The second eigenvector

图 3 柳杉树轮 $\delta^{13} \mathrm{C}$ 随方位变化的第一(左)、第二(右)典型场

Fig.3 The first (left) and second (right) eigenvectors of $\delta^{13} \mathrm{C}$ variation in the cryptomeria tree ring against azimuths

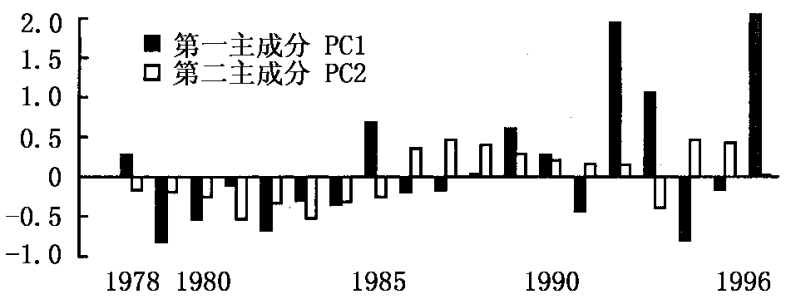

图 4 对应于第一、二典型场的时间系数 (主成分)

Fig.4 The first and second principle components

对应的主成分, 其中第一、二典型角分布及其主成 分, 分别代表了树轮 $\delta^{13} \mathrm{C}$ 随方位和时间变化方差的 $77 \%$ 和 $9 \%$ ，共计 $86 \%$ 。因此，近 19 年来 8 个方位柳
杉 $\delta^{13} \mathrm{C}$ 随时间与方位演变特征可用前两个典型角 分布 (图 3) 及其主成分 (图 4) 代表。而剩余的 6 个 累计方差占总体方差的 14\%，作为种种随机因素造 成的偏差而忽略不计。由图 3 可见，第一典型角分 布在各方位均为正值，代表各个方位一致的变化特 征,也就是说当某年第一主成分 (记为 PC1) 的值为 正时，代表所有方位 $\delta^{13} \mathrm{C}$ 均比相应方位多年平均值 大相反,PC1 为负则代表偏小。因此,PC1 代表了 树轮最主要的共同变化特征, 实际上也可以看作是 整个树轮 $\delta^{13} \mathrm{C}$ 平均值的年际变化特征。但同时也 可以看到这种变化量的大小在不同方位是不同的， 北 $(\mathrm{N})$ 、西 $(\mathrm{W})$ 、西南 $(\mathrm{SW})$ 、南 $(\mathrm{S})$ 方位 $\delta^{13} \mathrm{C}$ 年际变率 明显大于其它 4 个方位, 最大的为北 (N) , 最小的为 东 $(\mathrm{E})$ 。第二典型场在 N、W、SW、S 4 个方位为正， 其它 4 个方位为负。表明前 4 个方位与后 4 个方位 存在随时间相反的变化趋势。其中变率最大的为 $\mathrm{NW}$ 方位。因此, PC2 代表了这种差异的年际变化, 对应某一年的 PC2 值为正, 则该年前 4 个方位 $\delta^{13} \mathrm{C}$ 偏大, 后 4 个方位偏小, PC2 值的绝对值越大表示这 种差异越明显。那么, 是什么原因造成树轮 $\delta^{13} \mathrm{C}$ 年 际变率在不同方位的差异呢? 这可能仍是树木生物 化学过程对树木所处不同方位的环境条件差异的响 应。

从图 4 可以看出 ,PC1 近 19 年来总体是增大的 (与图 2 一致) ,1985 年以前大多数为负 (即小于 19 年的平均值) ,最小的为 1979 年, 1988 年以后大多数 年份为正 (大于 19 年的平均值), 最大为 1996 年。 PC2 在 1985 年及其以前多数为负, 1985 年以后除 1993 年为负外其余全为正。根据图 4 所示第一、二 主成分的正 $(+)$ 负 $(-)$ 号配置可将近 19 年 $\delta^{13} \mathrm{C}$ 的 方位分布演变分为 4 个基本形态。分类方法为 :型 $(+,+) 、$ 二型 $(+,-) 、$ 三型 $(-,+) 、$ 四型 $(-$, - ) 括号中的符号为第一、二主成分的符号。分类 结果如表 1 。

将各型所对应年份进行合成就可以得到 4 种 $\delta^{13} \mathrm{C}$ 角分布型。为了突出各分布型与多年平均状况 的差异 将这 4 种基本型与多年平均状况相减, 得到 各型 $\delta^{13} \mathrm{C}$ 角分布与平均状况的偏差, 如图 5 所示。

从图 5 可见，一型年份各方位 $\delta^{13} \mathrm{C}$ 较多年平均 偏大, 其中 $N 、 W 、 S W 、 S$ 较平均值偏大约 0.5 , 而其余 4 个方位偏大不到 0.2 。二型各方位也较多年平均 值偏大, 但各方位间差异不大, 除 $\mathrm{N}$ 方位较小外, 其 余均在 $0.2 \sim 0.4$ 之间。三型为各方位 $\delta^{13} \mathrm{C}$ 较平均状 况偏小，其中 NW、NE 方位约偏小 0.4 , 而其余方位 
偏小 $0.2 \sim 0.3$ 。四型表现为 NE、E、SE 与平均状况 一致,NW 方位较平均状况偏大约 $0.1, \mathrm{~N}$ 方位偏小 0.2 , 其余 3 个方位则偏小约 0.3 ,比较而言, 该型与 平均状况最接近。

表 $1 \quad \delta^{13} \mathrm{C}$ 角分布型年表

Table 1 The $\delta^{13} \mathrm{C}$ azimuth distribution types in the period of 1978-1996

\begin{tabular}{ccccccc}
\hline $\begin{array}{c}\text { 类型 } \\
\text { Type }\end{array}$ & \multicolumn{5}{c}{$\begin{array}{c}\text { 年份 } \\
\text { Year }\end{array}$} \\
\hline 1 & 1988 & 1989 & 1990 & 1992 & 1996 \\
2 & 1978 & 1985 & 1993 & & & \\
3 & 1986 & 1987 & 1991 & 1994 & 1995 & \\
4 & 1979 & 1980 & 1981 & 1982 & 1983 & 1984 \\
\hline
\end{tabular}

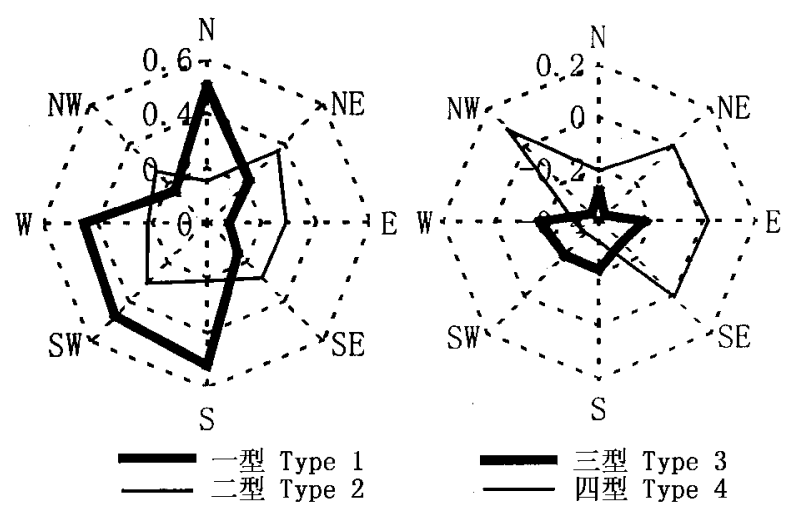

图 5 -、二(左)、三、四(右)型 $\delta^{13} \mathrm{C}$ 偏差方位分布图

Fig.5 The deviation of $\delta^{13} \mathrm{C}$ from its mean azimuth distribution for Type $1,2,3$ and 4

\section{3 柳杉 $\delta^{13} \mathrm{C}$ 角分布与环境因子的相关分析}

某一年出现哪一个 $\delta^{13} \mathrm{C}$ 角分布型, 是由与该年 对应的 PC1 和 PC2 的符号决定的。因此只要搞清 这两个主成分的变化特征及其与环境因子的关系, 就可以了解 $\delta^{13} \mathrm{C}$ 角分布的年际变化特征及其与环 境因子的关系。

采用公式 (7)分别计算了前期、同期气候因子与 PC1 和 PC2 的相关系数, 发现 PC1 与前一年 $8 \sim 12$ 月各月降水量都呈负相关关系, 其中与 12 月的相关 系数为 -0.47 ,与这 5 个月总降水量相关系数为 0.42 。与当年 1.3 月降水量相关系数为 0.31 和 0 . 58 ,与 $1 \sim 3$ 月总降水量显著正相关，相关系数为 0 . 59 通过信度为 0.01 的检验标准。而与当年其它月 的降水量相关不显著。这表明当前一年 $8 \sim 12$ 月降 水量偏少, 当年 $1 \sim 3$ 月降水偏多时, 树轮 $\delta^{13} \mathrm{C}$ 角分 布容易出现一、二型，即在各方位 $\delta^{13} \mathrm{C}$ 较平均状况 偏大。相反时，则易出现三、四型分布。PC2 与 7 月 降水量存在弱的负相关 $(-0.35)$,说明 7 月降水偏 多时, 树轮 $\delta^{13} \mathrm{C}$ 角分布异常易出现二、四两型, 否则
易出现一、三两型。总体而言,PC1 与前一年 8 月至 当年 3 月期间天目山地区降水状况关系密切, 而 PC2 则与降水量关系相对而言较差。

通过与气温的相关分析发现, PC1 与当年 3 7 月各月平均温度都呈现负相关, 其中与 7 月平均气 温的相关系数为 -0.39 ,与 $3 \sim 7$ 月 5 个月的平均气 温显著负相关, 相关系数为 -0.47 , 通过信度 0.05 的显著性检验 $(0.46)$ 。与 9 月平均气温正相关, 相 关系数为 0.39 。另外, 通过与同一时段的最高、最 低气温的相关分析, PC1 与它们有相同的相关关系。 $\mathrm{PC} 2$ 与月平均气温大多数为正相关, 其中与 1 月气 温相关系数为 0.42 ,与前一年 8 月至当年 1 月 6 个 月的平均气温显著正相关，相关系数高达 0.64 , 通 过信度为 0.01 的显著性检验 $(0.58)$ 。

根据 PC1、PC2 与各种角分布型的对应关系，以 及两个主成分与环境变量的相关性, 得到 4 种 $\delta^{13} \mathrm{C}$ 角分布型出现的适宜环境条件，如表 2 所示。

表 2 环境变量与 $\delta^{13} \mathrm{C}$ 角分布型之间的关系

Table 2 Relation between environmental state and $\delta^{13} \mathrm{C}$ azimuth distribution types

\begin{tabular}{|c|c|c|c|c|}
\hline \multicolumn{4}{|c|}{$\begin{array}{c}\text { 环境条件 } \\
\text { Environmental conditions }\end{array}$} & \multirow{2}{*}{$\begin{array}{c}\text { 分布型 } \\
\text { Type }\end{array}$} \\
\hline $\mathrm{R}_{8 \sim 12}$ & $\mathrm{R}_{1 \sim 3}$ & $\mathrm{~T}_{3 \sim 7}$ & $\mathrm{~T}_{8 \sim 1}$ & \\
\hline 少 Little & 多 More & 低 Low & $\begin{array}{l}\text { 高 High } \\
\text { 低 Low }\end{array}$ & $\begin{array}{l}\text { Type } 1 \\
\text { Type } 2\end{array}$ \\
\hline 多 More & 少 Little & 高 High & $\begin{array}{l}\text { 高 High } \\
\text { 低 Low }\end{array}$ & $\begin{array}{l}\text { Type } 3 \\
\text { Type } 4\end{array}$ \\
\hline
\end{tabular}

$\mathrm{R}_{8 \sim 12}$ : 前一年 8 月至 12 月降水总量 Total rainfall in the period of August to December in the previous year $\mathrm{R}_{1 \sim 3}$ : 本年度 1 至 3 月降水总 量 Rainfall in the period of January to March $\mathrm{T}_{3 \sim 7}$ : 本年度 3 至 7 月平 均气温 Averaged temperature in March to July $\mathrm{T}_{8 \sim 1}$ : 前一年 8 月至本年 1 月平均气温 Averaged temperature in the period from previous August to present January

\section{3 讨 论}

以上研究表明 ,天目山柳杉树轮 $\delta^{13} \mathrm{C}$ 沿不同方 位存在显著的差异，这种差异的平均状况可能是由 于树木生长环境在不同方向上的差异造成的 (Pieter \& Mook ,1980)。这种差异并不是一成不变的,而是 存在显著的年际变化, 并且在不同方位 ${ }^{13} \mathrm{C}$ 的变率 也不同。年际变化可以用 4 个基本角分布型的更替 来表征,也可以用第一、二主成分的年际变化来表 示。各方位变率的差异可能是树木因不同方位环境 条件的差异而对气候变化的生理反应不同造成的。 有些水、肥条件较差的方位反应敏感(变率大) ,而另 一些水、肥条件好的方位反应迟钝 (变率小)。在这 些环境条件中, 地理环境条件的变化比较小, 气候状 
况的年际变化明显，因此无论是 8 个方位 $\delta{ }^{13} \mathrm{C}$ 平均 值的年际变化, 还是角分布型的年际更替, 都可能是 气候变化造成的。对于前者大量研究成果已经证实 (Leavitt \& Long , 1984; 1989; 1991; 刘禹等, 1996 ;钱君 龙等 ,2001a）,而对于后者一些研究者仅提及这种现 象的存在 (Robertson et al.,1997 ; Leavitt \& Long， 1984) , 而对其进行深入研究的则不多。本文研究表 明, 不同的气候背景与不同的角分布型相联系。由 于天目山属亚热带湿润气候, 树木生长期长, 而且生 长旺盛, 物质输送能力强, 具有较强的营养储存能 力。因此 $\delta{ }^{13} \mathrm{C}$ 不但受当年气候的影响, 而且受前期 气候状况的影响 (Feng \& Epstein ,1995)。前一年秋 冬季节的降水和当年春夏季节的气温影响树轮 $\delta{ }^{13} \mathrm{C}$ 整体的变化(钱君龙等，2001a）,而前一年秋冬季节 的平均气温则影响其在各个方位增减的差异。

天目山地区, 雨季很长, 有充分的降水。在一定 的范围内, 能满足树木在生长旺盛期的各种生理活 动, 因此, $\delta^{13} \mathrm{C}$ 对当年 4 9 月该时段的降水量变化 反应不敏感。但当气候极端异常时，仍可能对其产 生影响，如 1994 年 $\delta^{13} \mathrm{C}$ 值是近 19 年中最低的，这可 能是因为该年 7 月降水量只有 $67 \mathrm{~mm}$,与同期多年 平均值 $244 \mathrm{~mm}$ 相比，减少 $72.5 \%$ 。

影响 ${ }^{13} \mathrm{C}$ 的因素很多, 本文的研究区域也不是 限制因子地区，环境因素对 $\delta^{13} \mathrm{C}$ 的影响机理很复 杂。另外，因客观原因，本文的研究资料仅有 19 年， 非常有限。因此本文仅从天目山这一湿润地区树轮 $\delta^{13} \mathrm{C}$ 角分布的存在性及其年际变化与环境因素的相 关性方面进行了研究, 研究结果还有待于积累更多 资料, 进一步进行论证。

\section{参 考 文 献}

Beerling, D. J. 1996. $\delta{ }^{13} \mathrm{C}$ discrimination by fossil leaves during the late-glacial climate oscillation 12-10 ka, BP: Measurements and physiological controls. Oecologia, 108:29 37 .

Feng, X.H. \& S. Epstein. 1995. Carbon isotopes of trees from arid environments and implications for reconstructing atmospheric $\mathrm{CO}_{2}$ concentration. Geochemica Cosmochimica Acta, 59: 2599 2608.

Jiang, G.M. (蒋高明), Y.X. Huang (黄银晓), G.J. Wan (万
国江) \& Y. C. Chen (陈业材). 1997. A study on the $\delta^{13}$ C values of tree rings and their indicative functions in revealing atmospheric $\mathrm{CO}_{2}$ changes in north China. Acta Phytoecologica Sinica (植物生态学报), 21: 155 160. (in Chinese with English abstract)

Leavitt, S. W. \& A. Long. 1984. Sampling strategy for stable carbon isotope analysis of tree in pine. Nature, 311: 145 147 .

Leavitt, S. W. \& A. Long. 1989. Drought indicated in carbon13/carbon-12 rations of southwestern tree rings. Water Resources Bulletin, 25: $341 \sim 347$.

Leavitt, S. W. \& A. Long. 1991. Seasonal stable-carbon isotope variability in tree rings: possible paleoenvironmental signals. Chemical Geology (Isotope Geoscience Section), 87: 59 70 .

Liang, E.Y. (梁尔源), X.M. Shao(邵雪梅) , Y.X. Hu(胡玉 喜) \& J. X. Lin (林金星). 2001. Variation in tree ring growth indices of Picea meyeri from the sandy land in the steppe of Inner Mongolia. Acta Phytoecologica Sinica (植物生态学报), 25: $190 \sim 194$. (in Chinese with English abstract)

Liu, Y. (刘禹), D. X. Wu (吴定祥), S. W. Leavitt \& M. K. Hughes. 1996. The $\delta{ }^{13} \mathrm{C}$ values of tree rings and climate change in Huanglin county. Science in China (Series D) (中国科学 D 辑), 26: $125 \sim 130$. (in Chinese with English abstract)

Qian, J.L. (钱君龙), J. Li (李军), Q.P. Tu(屠其璞) \& S. M. Wang (王苏民). 2001a. Reconstruction of the last $160 \mathrm{a}$ climate in Tianmu Mountain regions by the $\delta{ }^{13} \mathrm{C}$ values of tree rings. Science in China (Series D) (中国科学. D 辑), 31: $333 \sim 341$. (in Chinese with English abstract)

Qian, J.L. (钱君龙)，Z.W. Deng (邓自旺)，Q.P. Tu (屠其 璞) S. M. Wang (王苏民) \& Y. S. Huang (黄耀生). 2001b. Climatic significance of the $\delta{ }^{13} \mathrm{C}$ time series in tree rings from Tianmu Mountain. Science in China (Series D) (中国科学 D 辑), 44: 1140 1146. (in Chinese with English abstract)

Robertson, I., V. R. Switsur \& A. H. C. Cater, 1997. Signal strength and climate relationships in ${ }^{13} \mathrm{C} /{ }^{12} \mathrm{C}$ rations of tree ring cellulose form oak in east England. Journal of Geophysical Research, 102(D16) : $507 \sim 516$.

Schleser, G. H. 1995. Parameters determining carbon isotope ratios in plants, in Palaokimaforschung 15. In: Frenzel, B., B. Stauffer \& M. M. Weiss eds. European Scientific Found, France. 71 $\sim 96$.

Schleser, G. H., G. Helle, A. Lücke \& H. Vos. 1999. Isotope signals as climate proxies: the role of transfer functions in the study of terrestrial archives. Quaternary Science Reviews, 8: 927 $\sim 943$.

Sheu, D.D. \& C.H. Chiu. 1995. Evaluation of cellulose extraction procedures for stable carbon isotope measurement in tree ring research. International Journal of Environmental Analysis in Chemistry, 59: $59 \sim 67$.

Tans, P. P. \& W. G. Mook. 1980. Past atmospheric $\mathrm{CO}_{2}$ levels and the ${ }^{13} \mathrm{C} /{ }^{12} \mathrm{C}$ ratios in tree rings. Tellus, 32: $268 \sim 283$.

Tu, Q.P. (屠其璞), J.D. Wang (王俊德) \& Y.G. Ding (丁裕 国). 1984. Applying probability and statistics in meteorology. Beijing: China Meteorology Press. $165 \sim 183$. (in Chinese with English abstract) 